\title{
Liquid metal microcoils for sensing and actuation in lab-on-a-chip applications
}

\author{
Tian Fook Kong • Nam-Trung Nguyen
}

Received: date / Accepted: date

\begin{abstract}
The use of metals and alloys with melting point near room temperature, called here as liquid metals, allows the integration of complex three-dimensional metallic micro structures in lab-on-chip devices. The process involves the injection molten liquid metal into microchannels and subsequent solidification at room temperature. The paper reports a technique for the fabrication of threedimensional multilayer liquid-metal microcoils by lamination of dry adhesive films. The adhesive-based liquid metal microcoil could be used for magnetic resonance relaxometry (MRR) measurement in a lab-on-a-chip platform. Not only that the coil has a low direct-current resistance, it also has a high quality factor. In this paper, we investigate the sensing and actuating capabilities of the liquid metal microcoil. The sensing capability of the microcoil is demonstrated with the coil working as a blood hematocrit level sensor. In a magnetic resonance relaxometry measurement, the transverse relaxation rate of the blood sample increases quadratically with the hematocrit level due to higher magnetic susceptibility. Furthermore, a vibrating adhesive membrane with the embedded coil was realized for electromagnetic actuation. A maximum deflection of approximately 50 microns at a low resonance frequency of $15 \mathrm{~Hz}$ can be achieved with a maximum driving current of $300 \mathrm{~mA}$.
\end{abstract}

Keywords liquid metal $\cdot$ microcoils $\cdot$ magnetic resonance relaxometry $\cdot$ vibrating membrane $\cdot$ micropump

\footnotetext{
Tian Fook Kong

School of Mechanical and Aerospace Engineering,

Nanyang Technological University,

50 Nanyang Avenue, 639798, Singapore.

Nam-Trung Nguyen

Queensland Micro and Nanotechnology Centre,

Griffith University,

170 Kessels Road, Brisbane, 4111, Australia.

E-mail: nam-trung.nguyen@griffith.edu.au
} 


\section{Introduction}

The introduction of microsolidics [1] opened up a new way for the integration of electrical conductors in lab-on-a-chip (LOC) devices. The fabrication process starts with the injection of a liquid metal that has low melting temperature, such as indium, gallium, and tin, at elevated temperature into a microchannel. The metal is subsequently allowed to cool and solidify to form an embedded electrical conductors. The unique feature of this method is the implementation of flexible complex three-dimensional metallic microstructures with a minimum fabrication effort. The mechanically deformable liquid-metal structures have found new applications such as pressure sensor [2] and tunable fluidic antenna [3]. The main advantages of liquid metal technology over other conventional microfabrication techniques are the low direct current resistance, low fabrication cost, and high aspect ratio. Conventional fabrication techniques have their respective problems. For instance, microcontact printing [4] has a high contact resistance. Electroplating [5] has a porous and rough surface finish. Focused ion milling [6] requires an expensive fabrication facility and a corresponding high cost. E-beam and thermal evaporation [7] has low material deposition rate leading to a low aspect ratio.

Recently, we have reported a novel technique for the fabrication of threedimensional multilayer liquid-metal microcoils by lamination of dry adhesive films [8]. The liquid metal microcoil was used as the receiver coil for magnetic resonance relaxometry (MRR) measurement in a lab-on-a-chip platform. The coil not only has a low direct-current resistance, but also a high quality factor. In this paper, we further investigate the sensing and actuating capabilities of a liquid-metal microcoil as a sensor as a hematocrit level in blood and an actuating membrane for possible use in a micropump. The hematocrit level of human blood is the volume percentage of red blood cells in blood, and varies between $40-50 \%$ for a healthy individual. If the red blood cell count (RBC) or the hematocrit level is low, the individual is diagnosed as anemic. Low RBC count, also termed as anemia hypoxia, could be lethal as the main function of $\mathrm{RBC}$ is to supply oxygen to the body. Centrifugation and optical method are commonly used in hospital for the determination of blood hematocrit level. However, these methods are difficult to be integrated in a lab-on-chip platform. In contrast, the liquid metal microcoil investigated here could detect a subtle change in the blood hematocrit level by monitoring the transverse relaxation rate, $R_{2}$ using MRR measurement. The relaxation rate $R_{2}$ of a blood sample is found to be increasing as a square function of the blood hematocrit level with a goodness of fit of more than 0.99. Hence, the liquid metal microcoil, which readily integrates with lab-on-chip, could detect a shift in the blood hematocrit level with high sensitivity.

On the other hand, we further demonstrate the actuation capabilities of the liquid metal microcoil embedded in the adhesive membrane as an actuator with possible use in a micropump. Although external macroscale pumps such as syringe and peristaltic pumps are usually used in laboratory experiments for routine dispensing of fluids, the development of miniaturized pumps has taken off with the advancement in the lab-on-chip technology. Self-contained micropumps are preferred for manipulating a minute amount of fluid with high precision in a microfluidic systems [9]. Lab-on-a-chip applications often require the manipulation control of of fluid volume on the order of $1 \mathrm{pL}$ [10-12]. To avoid dead volume, a micropump should be integrated on the same chip with other components. Thus, 
the actuating membrane is preferably integrated with the pumping chamber on the same lab-on-a-chip platform. Lamination of stacked adhesive layers would allow the implementation of both sensing and actuating coils as well as other microfluidic components on the same device. A driving alternating current applied to the liquid-metal microcoil placed next to a permanent magnet causes the membrane to oscillate at the frequency of the current due to the induced magnetic force. The actuating membrane will be characterized and evaluated for micropump applications. The advantage of this actuating membrane is its flexibility and low resonant frequency. Since many micropumps are working at pumping cycles on the order of few hertz, a soft pump membrane would be ideal. Furthermore, the resonant frequency of the membrane could be adjusted by varying the membrane thickness, material, size and geometry.

\section{Materials and methods}

\subsection{Device fabrication}

Multiple layers of laser-cut adhesive films (Arclad 5913 Adhesives Research Inc., USA) were laminated to form a flexible membrane with an embedded microcoil. The $75 \mu \mathrm{m}$ thick adhesive film is made of polyester coated with acrylic adhesive on both sides. The lamination approach allows the layers to be fabricated easily by laser machining, without the need for photolithography and clean room access. The microchannel layouts were designed using Autocad (Autodesk, USA) and engraved with a laser cutting machine (M300, Universal laser system Inc, USA). The microcoil was made of three stacked layers: the channel layer, the interconnect layer, and the lead-out layer (Fig.1(a)). The microcoil used for the MRR sensing has 5 turns and a feature size of $250 \mu \mathrm{m}$, while the microcoil used for actuation has 10 turns and a feature size of $1 \mathrm{~mm}$ for both the coil width and gap. The adhesive stack is placed on a printed circuit board with contact pads and corresponding through holes for the injection of liquid metal. Liquid metal gallium (Sigma-Aldrich, USA) with a low melting point of $29.8{ }^{\circ} \mathrm{C}$ was used as the conducting material with a electrical resistivity of $270 \mathrm{n} \Omega \cdot \mathrm{m}$. First, gallium was heated in an oven at $60{ }^{\circ} \mathrm{C}$ for 10 minutes. Subsequently, the molten gallium was injected into the spiral channel through one of the metal contact pads on the printed circuit board (PCB) with a drilled through hole of $1 \mathrm{~mm}$ in diameter (Fig.1(b). The flow of the liquid metal continues to the lead-out layer via the middle inter-connect layer that has a laser-cut through hole. Finally, the molten gallium metal exits through the lead-out layer on the other side of the stack to the drilled through hole on the other metal contact pad of the PCB. The microcoil was then allowed to cool at room temperature for about an hour to make sure that the liquid gallium has sufficient time to solidify.

To precisely align and bond the adhesive layers, each layer were designed with 2-mm alignment holes at its four corners. The adhesive layers were guided and bonded onto each other with the help of four alignment pins made of blunt-head needles (G15, Nordson EFD Inc., USA). The alignment accuracy ranges approximately between 50 to $100 \mu \mathrm{m}$. The typical direct current resistance of the microcoil is $0.3 \Omega$. The large contact area between the liquid metal and the contact pads on the PCB allows an excellent electrical connection to external devices. However, if 
a)

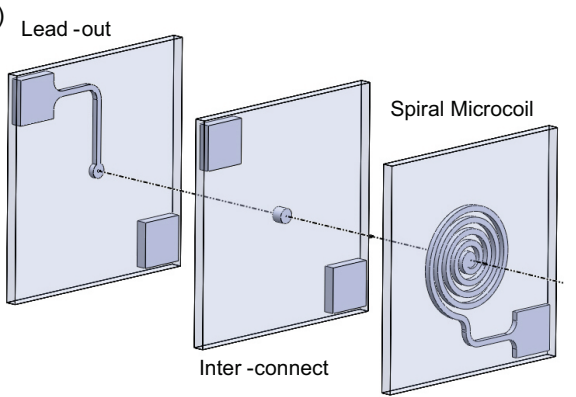

b)

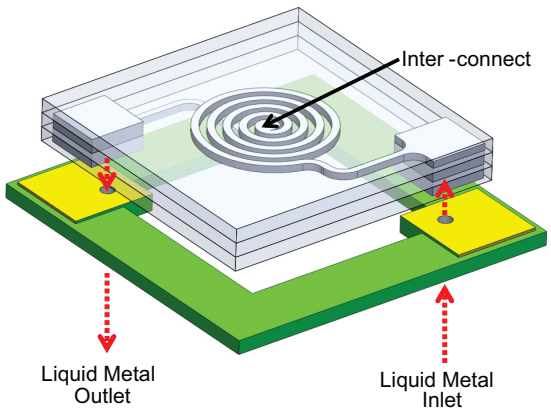

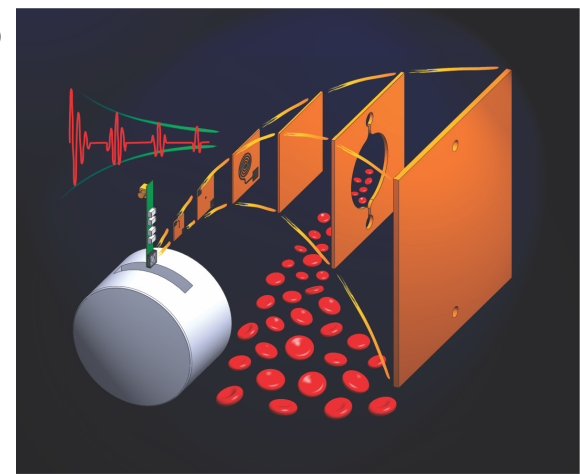

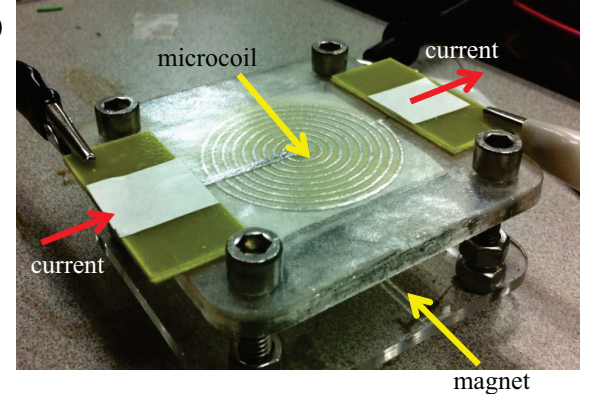

Fig. 1 Fabrication of liquid-metal microcoils for sensing and actuation: (a) The microcoil consists of three stacked layers: spiral channel, inter-connect, and lead-out; (b) The liquid metal is injected to the spiral coil via a mechanically drilled hole on a pad on the PCB. The flow continues to the lead-out layer via a middle inter-connect layer and finally exits through the other metal contact pad on the PCB. The microcoil used for MRR sensing has 5 turns and a feature size of $250 \mu \mathrm{m}$, while the microcoil used for actuation has 10 turns and a feature size of $1 \mathrm{~mm}$ for both the coil width and gap; (c) Schematic diagram of the staking approach for the fabrication of multilayered microcoil with integrated microfluidic chamber for MRR blood hematocrit level sensing in a lab-on-a-chip setting; (d) Image of the actuation membrane with embedded liquid-metal coil.

the inter-connect layer is misaligned with the spiral and lead-out layers, the microcoil will either has a high resistance or becomes an open circuit. In order to achieve a high-aspect-ratio microcoil, five layers of adhesive film were laminated together prior to laser cutting, giving a coil thickness of $375 \mu \mathrm{m}$. For sensing application, the coil stack is attached to a sample chamber made by the same lamination approach. The schematic diagram shown in Fig.1(c) illustrates the staking approach for the fabrication of multilayered microcoil with integrated microfluidic chamber for MRR blood hematocrit level sensing. Blood sample was loaded in the microfluidic chamber stacked on top of the liquid metal microcoil, and the integrated device is placed in a $0.5 \mathrm{~T}$ permanent magnet for MRR measurements. The image of the actuation diaphragm with liquid metal microcoil embedded is shown in Fig.1(d). 


\subsection{Experimental setups}

\subsubsection{Blood hematocrit sensor}

The liquid-metal microcoil was used as the receiver for MRR measurements of blood samples. The laminated adhesive device was integrated with a nuclear magnetic resonance (NMR) probe for measuring the $0.5 \mathrm{~T}$ proton ${ }^{1} \mathrm{H}$ transverse relaxation rate. The resonant frequency of the probe was tuned and matched to $21.65 \mathrm{MHz}$ with capacitors in a simple L-network configuration. The tuning and matching capacitance required were $1000 \mathrm{pF}$ and $220 \mathrm{pF}$, respectively. The probe was placed in a $0.5 \mathrm{~T}$ NMR permanent magnet (PM-1055, Metrolab Instruments, Switzerland) and connected to a commercial bench-top NMR spectrometer (KEA2, Magritek, New Zealand) via a RF coaxial cable. The NMR spectrometer has a maximum power output capacity of $100 \mathrm{~W}$ and the software (Prospa v2.0) provided by the system manufacturer was used to capture and process the NMR data. The NMR pulse duration and amplitude for the $90^{\circ}$ pulse used were $131 \mu \mathrm{s}$ and -30 dB (1.56 W) [8]. The Carr-Purcell-Meiboom-Gill (CPMG) spin echo pulse sequence was used to determine the $R_{2}$ time constant of the exponential decay of the voltage induced due to the dephasing and refocusing of the nuclei spin, following the repeated application of $180^{\circ}$ pulses after the initial $90^{\circ}$ pulse to tip the magnetization vector into the transverse plane.

\subsubsection{Actuating membrane}

In order to generate an alternating current from a direct current source, we built an electronic control circuit with a miniature electromagnetic relay (Fujitsu, Takamisawa RY-5WFZ-K). The relay has a maximum current rating of $2 \mathrm{~A}$ and requires a power up voltage of $5 \mathrm{~V}$. The relay works as an electrically operated switch. The on/off states of the relay is controlled by a transistor (TIP122 NPN) and a function generator (Agilent 33120A, USA). The frequency of the alternating current can be precisely controlled by changing the frequency of the input triggering square-wave signal from the function generator. Fig.2(a) shows the circuit diagram for the control electronics. The transistor acts as a switch that control the power supply to the relay. When the trigger signal to the transistor is in the "on" state, the relay is turned on. Terminal A-G and F-H are now connected, while terminal A-B and F-E become open. The current flows from the source to point A, G, D, $\mathrm{C}, \mathrm{H}$, and finally to the ground through point $\mathrm{F}$. On the other hand, the direction of the current supply to the microcoil is reversed when the triggering signal is in the "off" state. The relay is switched off and connections at terminal A-B and F-E are restored. Current flows from the source to point A, B, C, D, E, F, and finally to the ground. With such a configuration, the direction of the current supply to the microcoil is reversed every time the triggering signal changes from "on" to the "off" state, and vice versa. The change of the actuating current allows the membrane to deflect to both sides of it initial neutral position.

The interaction between a magnetic field and the alternating current passing through the microcoil induces a force leading to the vibration of the membrane. The $5 \mathrm{~cm} \times 5 \mathrm{~cm}$ square membrane is placed next to an axially magnetized nickelplated permanent magnet of 1 " in diameter and 1" thickness (DX0X0-N52, K\&J Magnetics Inc., USA) (Fig.2(b)). The grade N52 neodymium (NdFeB) magnet has 
a)

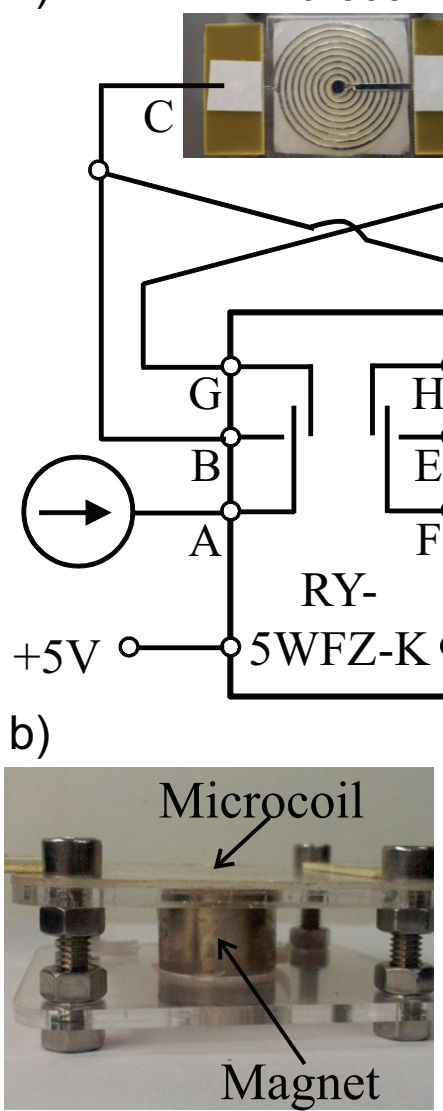

c)

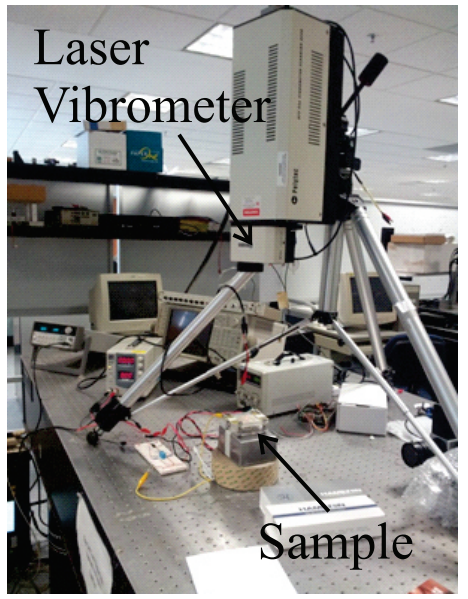

Fig. 2 The experimental setup for the actuating membrane with embedded liquid-metal coil: (a) Circuit diagram of the switching circuit for providing the microcoil with an alternating current. With the help of a function generator, the relay is triggered on and off to reverse the direction of the current supply to the coil. The vibration frequency of the membrane is determined by the frequency of the square-wave triggering signal; (b) A magnet is placed below the microcoil to provide a magnetic induction force when an alternating current passes through the microcoil. (c) The scanning laser vibrometer PSV-300 was used to investigate the characteristics of the vibrating membrane.

a surface field of $0.669 \mathrm{~T}$. When an alternating current flows through the liquid metal microcoil embedded in the adhesive membrane, the magnetic induction force changes its direction when the direction of the current is reversed. Therefore, the membrane oscillates upward and downwards in a periodic manner, following the excitation frequency of the alternating current. The vibrational characteristics of the membrane is studied with a scanning laser vibrometer (Polytec PSV-300, Germany). Fig.2(c) shows the experimental setup. The membrane and magnet was placed on an optical table, while the laser light from the laser vibrometer was focused onto the center of the coil. The signal acquired from the laser vibrometer was processed with the software supplied by Polytec. The time domain signal is 
fast Fourier transformed to study the frequency characteristics of the membrane. A parametric study was carried out to investigate the effect of the amplitude and frequency of the applied current on the vibration of the membrane.

\section{Results and discussions}

\subsection{Blood hematocrit sensor}

The hematocrit of a blood sample is defined as the ratio of the packed red blood cell volume to the total volume of the blood. In a healthy individual, the hematocrit levels for newborn baby ranges from $44 \%$ - $64 \%$, while adult male ranges from $42 \%$ - $52 \%$, and adult female $36 \%-48 \%$ [13]. If the red blood cell count or the quantity of hemoglobin is significantly lower than the normal range for a healthy individual, the person is diagnosed as anemic. Anemia hypoxia is a medical condition whereby the body lack oxygen due to the low red blood cell count [14]. As one of the main function of $\mathrm{RBC}$ is to transport oxygen from the lung to other parts of the body, the situation could be lethal if a person suffers from critically low RBC count. Therefore, access to fast, sensitive, and reliable diagnostic tool is of inevitable importance. Prompt diagnosis and timely treatment for anemia require a device that could detect the subtle change in the blood hematocrit level. Conventionally, the techniques used for blood hematocrit level identification include centrifugation, ultrasound [15], and optical method [16]. In this work, we carried out a parametric study on the relationship between the transverse relaxation rate of human red blood cell with its hematocrit level with the liquid metal microcoil. A portable MRR device could be developed in the future for anemia diagnosis.

Human blood samples were provided by Singapore - MIT Alliance for Research and Technology (SMART) Center (Infectious Disease IRG). The heparinized blood samples were washed and mixed with phosphate buffered saline (PBS $1 \mathrm{X}$ ) pH 7.4 (17-516Q, Lonza Walkersville, Inc., USA) at different volume ratios for preparing samples at various hematocrit levels. The total blood volume required for each measurement was $20 \mu L$, with $5 \mu L$ being used for MRR measurements in the sample chamber of the sensor and the remaining $15 \mu L$ being used for hematocrit level measurement using centrifugation. The samples were drawn into microcapillary tubes with inner and outer diameters of $550 \mu \mathrm{m}$ and $900 \mu \mathrm{m}$, respectively (Drummond Scientific Co., USA) by capillary filling. The tubes were then sealed with a vinyl-plastic compound (Critoseal, Krackeler Scientific, USA) and centrifuged at $6,000 \mathrm{~g}$ for 5 minutes (Sorvall Legend Micro 21, Thermo Scientific, USA).

A blood sample with higher hematocrit level has a higher magnetic susceptibility, which in turn increases the transverse relaxation rate. The detection of a subtle increase in the transverse relaxation rate due to the higher red blood cell count forms the basis of the MRR hematocrit detection. The relationship between the transverse relaxation rate of the blodd sample $R_{2}$ and the hematocrit level is shown in Fig.3. For each hematocrit level, the measurements were repeated five times. The error bars shows the standard deviation of the repeated measurements. The results clearly show that the transverse relaxation rate $R_{2}$ of the blood sample increases quadratically with the hematocrit level. The quadratic curve has a goodness of fit better than 0.99 . 


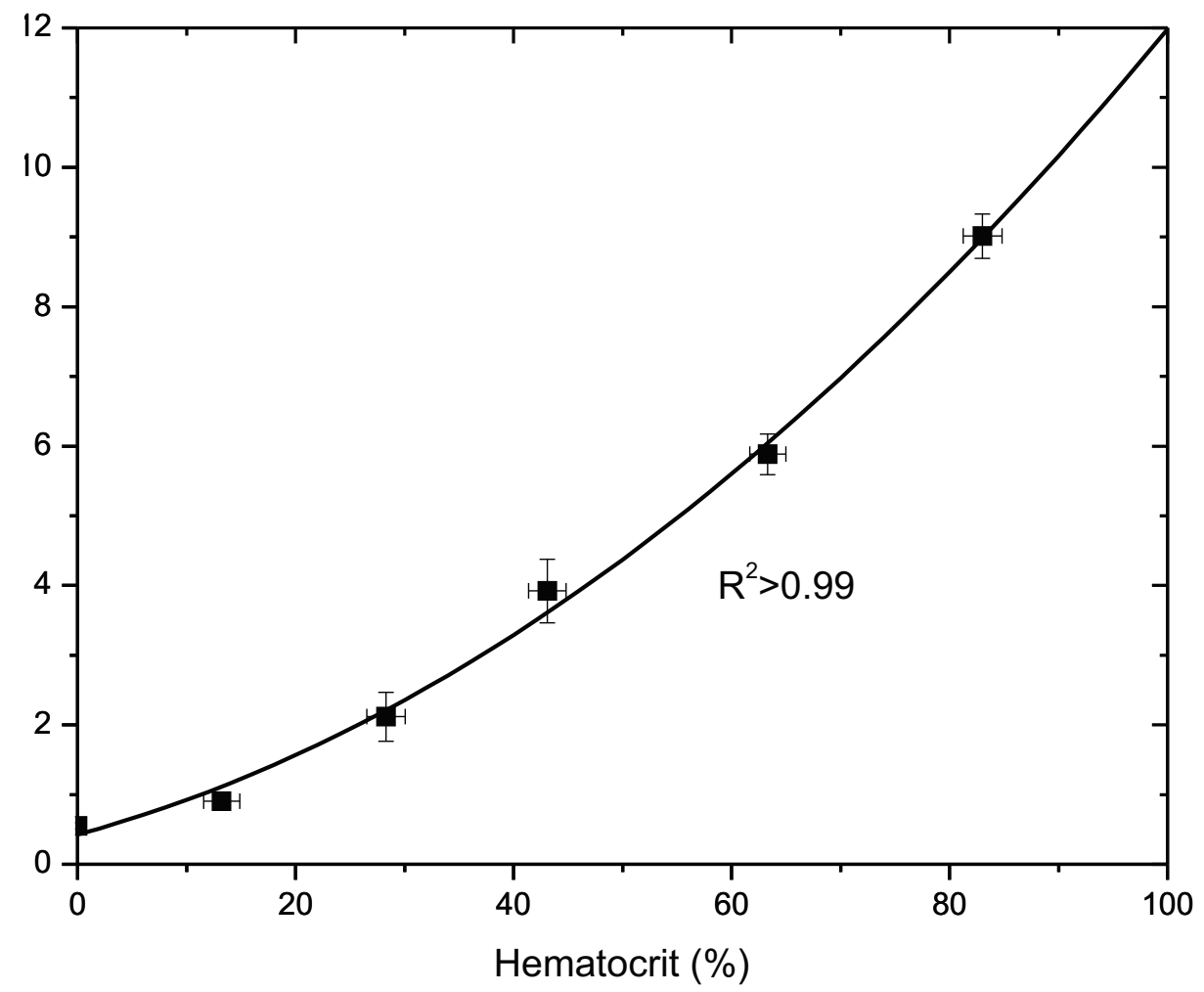

Fig. 3 The relationship between blood hematocrit level with the transverse relaxation rate, $R_{2}$. The transverse relaxation rate increases quadratically with the hematocrit level of the blood.

\subsection{Actuating membrane}

The magnetic induction force between the current passing through the liquid metal microcoil and the external magnetic field from the permanent magnet pulls the membrane closer to the magnet for one half a cycle of the alternating current and pushes the membrane away from the magnet for the other half cycle. Fig.4(a) shows the velocity of the membrane captured by the laser vibrometer. The amplitude and frequency of the alternating current supplied to the liquid metal microcoil were $0.3 \mathrm{~A}$ and $10 \mathrm{~Hz}$. The velocity of the membrane increases drastically when the current changes its direction. When the membrane is pushed away from the magnet, the velocity of the membrane is increased instantaneously in the positive direction and decays exponentially over time. Subsequently, when the current is reversed, the membrane is pulled towards the magnet. The membrane's velocity points to the negative direction and decays exponentially over time. The cycle of pushing and pulling the membrane causes the membrane to oscillate at its resonant frequency. In order to determine the amplitude of the membrane displacement, the velocity signal was integrated over time. The displacement-time graph is shown in 
Fig.4(b). The peak-to-peak amplitude of the membrane displacement is approximately $55 \mu \mathrm{m}$ for an input current of $0.3 \mathrm{~A}$ and $10 \mathrm{~Hz}$.

The resonance frequency of the vibrating membrane plays a pivotal role in determining its actuating performance. Fig.5(a) shows the zoom in of the exponential damping curve of the membrane when subjected to a switch in the current direction. The damped oscillation takes approximately $350 \mathrm{~ms}$ to complete 6 cycles of oscillations, before the oscillation diminishes of completely. A fast Fourier transform is applied to the damping oscillation signal shown in Fig.5(a) to determine the resonance frequency of the membrane. Fig.5(b) shows the frequency response of the membrane for input alternating currents of $0.1,0.2$, and $0.3 \mathrm{~A}$. There are 3 resonant peaks for the membrane at $15.6 \mathrm{~Hz}, 141 \mathrm{~Hz}$, and $188 \mathrm{~Hz}$. Since there is no shift in the frequency response for the three different input currents, the resonant frequency of the vibrating membrane is independent of the magnitude of the alternating current applied. However, the amplitude of the vibrating membrane is affected by the magnitude of the applied alternating current. The higher the input current, the higher is the amplitude of the frequency response. The resonant peak at $15.6 \mathrm{~Hz}$ is the lowest resonant frequency that corresponds to the fundamental mode shape $(1,1)$ of the vibrating membrane. The vibrating membrane could be optimized for different actuation applications. The resonant frequency of the membrane could be altered by manipulating the membrane thickness, material, size and geometry. When subjected to a uniform pressure $P$, the resonant frequency of a vibrating square membrane of side $a$ is given by [17]:

$$
f_{0}=\frac{1.654 t}{a^{2}} \sqrt{\frac{E}{\rho(1-\nu)^{2}}},
$$

where $t$ is the thickness, $E$ is the material Young's modulus, $\rho$ is the density, and $\nu$ is the Poisson's ratio. Therefore, the natural frequency of the membrane can be increased by either by reducing the membrane diameter or increasing its thickness by stacking more layers of adhesives.

Fig.6 shows the relationship between displacement amplitude of the vibrating membrane and the frequency and magnitude of the applied alternating current to the microcoil. The displacement amplitude is defined as half the peak-to-peak amplitude of the membrane vibration obtained from the time integration of the time-velocity signal. The amplitude of the membrane displacement is linearly proportional to the magnitude of the current applied regardless of the actuation frequencies, with goodness of fit better than 0.99. For example, for $1-\mathrm{Hz}$ oscillation, the displacement amplitude of the membrane increases from $7.74 \mu \mathrm{m}$ to $48.8 \mu \mathrm{m}$ when the applied AC current is increased from $0.05 \mathrm{~A}$ at $0.3 \mathrm{~A}$. The induced magnetic force, and thus the membrane displacement amplitude, increases as the magnitude of the applied current is increased. The maximum deflection of the membrane when subjected to a uniform pressure $P$ is given as[17]:

$$
\Delta_{\max }=0.001265 \frac{P a^{2}}{D}
$$

where $D$ is the flexural rigidity, a measure of stiffness of the membrane to bending:

$$
D=\frac{E t^{3}}{12\left(1-\nu^{2}\right)} .
$$



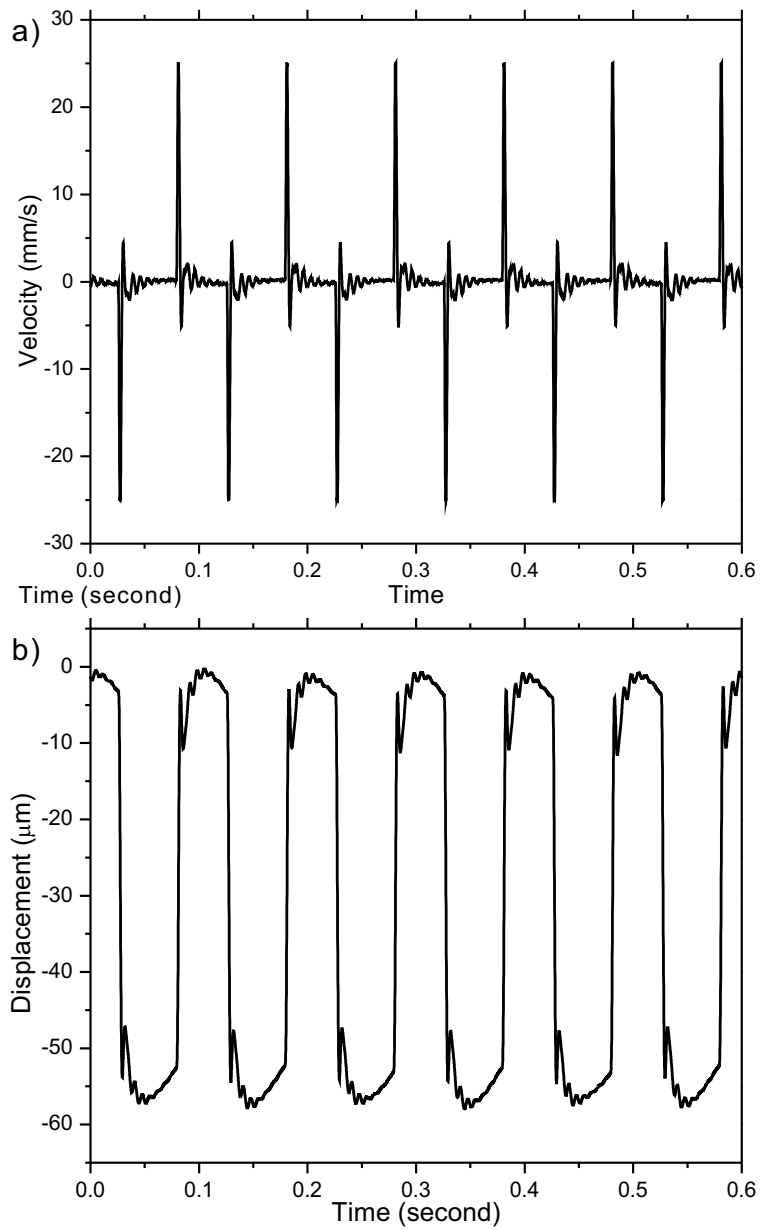

Fig. 4 (a)Velocity-time graph for the membrane captured with the laser vibrometer. The amplitude and frequency of the alternating current supplied to the microcoil are $0.3 \mathrm{~A}$ and $10 \mathrm{~Hz}$. The membrane accelerates in the positive and negative direction during the instantaneous change of direction in the current flow of the coil, and, subsequently, the velocity decays exponentially. (b) The amplitude-time graph obtained by integrating the velocity signal with time. The membrane exhibits a peak-to-peak displacement of approximately $55 \mu \mathrm{m}$ for the particular current amplitude and frequency.

The maximum deflection amplitude is proportional to the $a^{2} / t^{3}$ of the membrane. However, if the natural frequency of the membrane is increased by reducing the membrane diameter or increasing its thickness, the maximum deflection of the membrane is decreased at the same time. Therefore, it is a trade off between achieving higher resonant frequency or larger stroke for the pump membrane. 

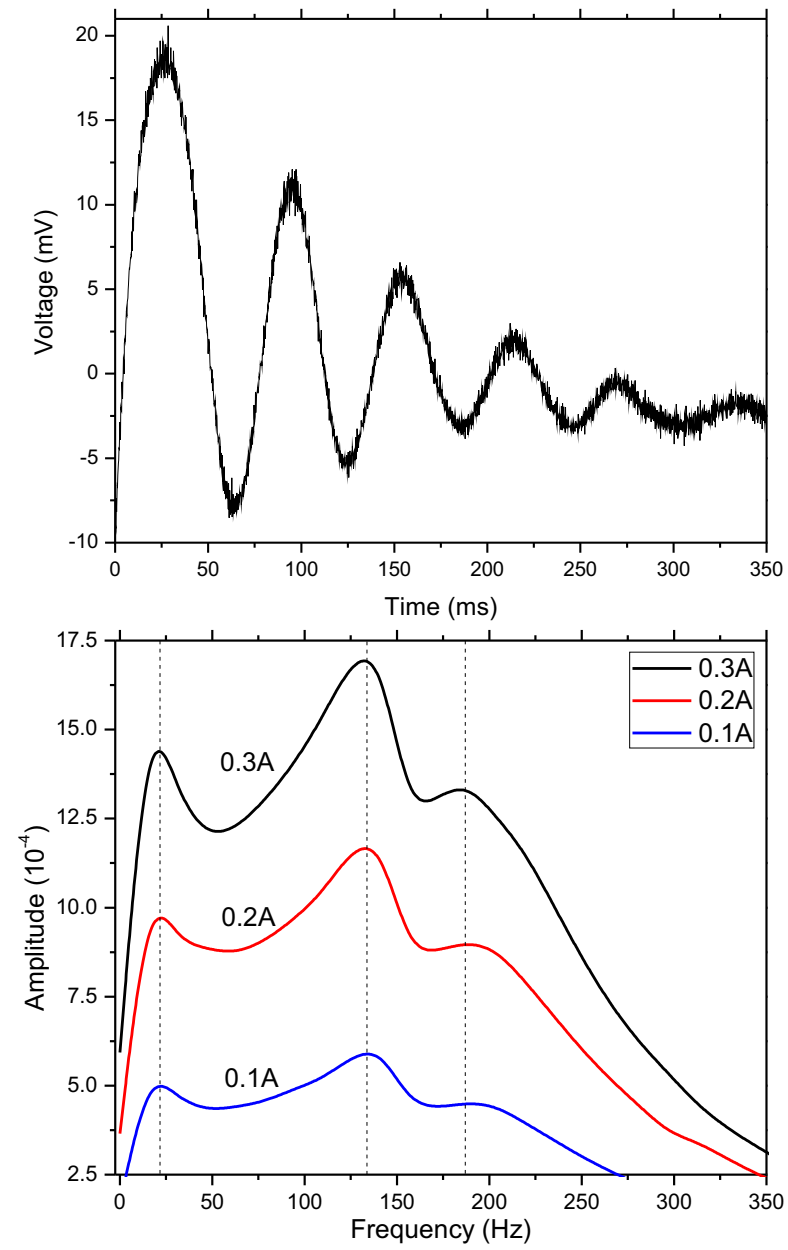

Fig. 5 (a) The exponential damping curve of the membrane when subjected to a switch in the current direction. (b) The frequency response of the membrane. There are 3 resonant peaks for the membrane at $15.6 \mathrm{~Hz}, 141 \mathrm{~Hz}$, and $188 \mathrm{~Hz}$.

\section{Conclusions}

In conclusion, we have successfully demonstrated the sensing and actuation capability of the three-dimensional multilayer liquid metal microcoils fabricated by lamination of dry adhesive sheets. The sensing capability of the liquid metal microcoil is demonstrated with the coil functioning as a blood hematocrit meter. The blood transverse relaxation rate increases quadratically with the hematocrit level. A portable MRR device could be developed in the future to aid the anemia diagnosis. Furthermore, the actuation capability of the microcoil is demonstrated 


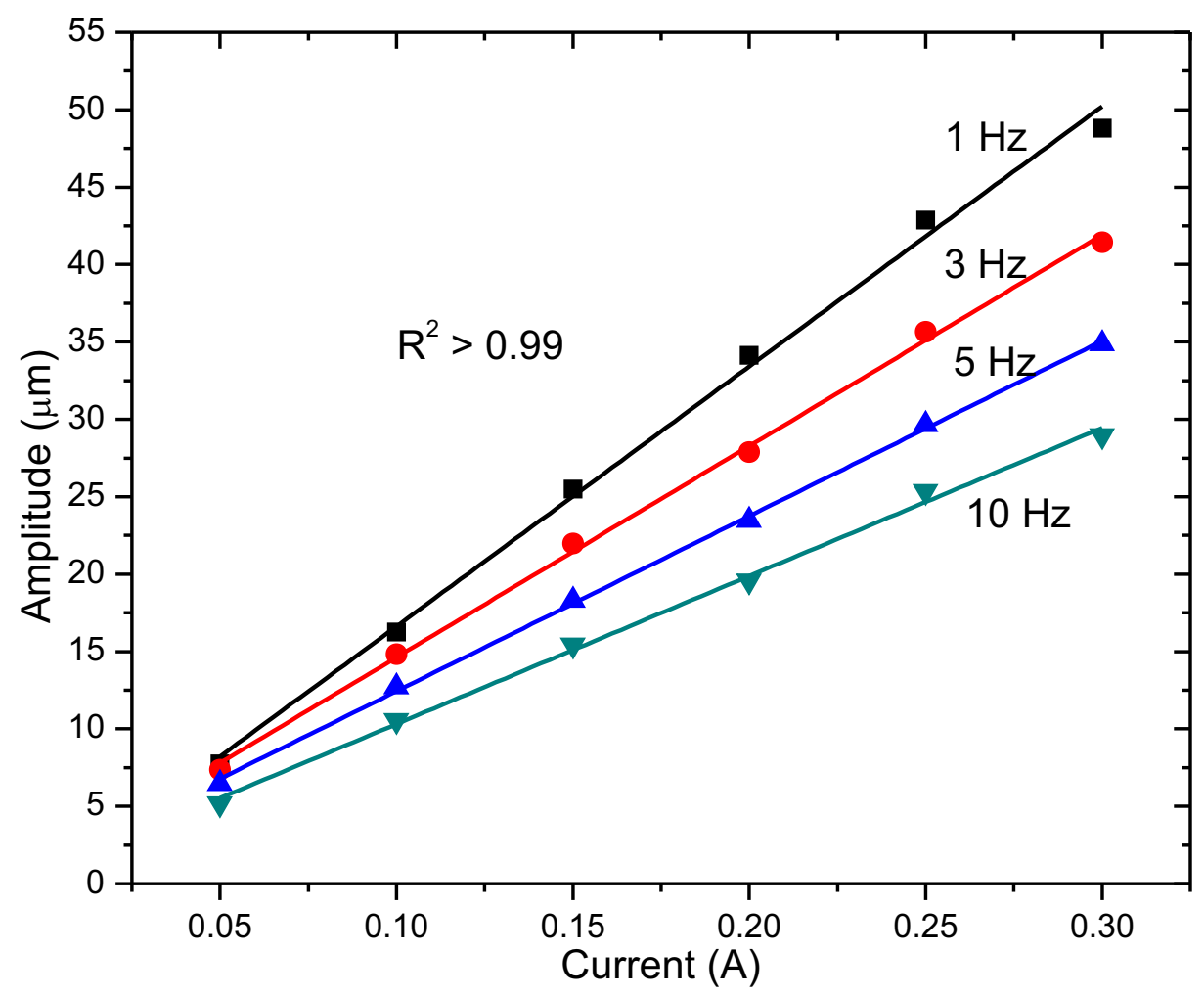

Fig. 6 Membrane's displacement amplitude vs magnitude of applied alternating current to the microcoil. The amplitude of the membrane displacement increases linearly with the magnitude of the current applied regardless of the actuation frequencies, with goodness of fit of greater than 0.99. As the excitation frequency is increases from $1 \mathrm{~Hz}$ to $10 \mathrm{~Hz}$, the amplitude of displacement decreases.

with the use of the laser-cut adhesive membrane with liquid metal microcoil embedded as the actuation diaphragm for an electromagnetic miniature pump. The interaction between the alternating current applied to the microcoil and the magnetic field from a permanent magnet placed in close proximity induces a magnetic force and causes the membrane to oscillate. The vibrational characteristics of the membrane such as the oscillation amplitude, velocity, and frequency response were investigated with a scanning laser vibrometer. The liquid metal microcoil investigated in this work has 10 turns and a feature size of $1 \mathrm{~mm}$ for both the coil width and gap. The amplitude of the membrane displacement could go as high as $50 \mu \mathrm{m}$ for an input current of $0.3 \mathrm{~A}$. The main advantage of using the membrane with embedded liquid-metal coil is that the membrane could be easily integrated with the pumping chamber in lab-on-a-chip platform. Furthermore, the membrane could be optimized for different application requirements by tuning the membrane thickness, material, size and geometry to achieve desired amplitude and frequency. 


\section{Acknowledgments}

This work is supported by Singapore - MIT Alliance for Research and Technology (SMART) Centre, BioSystems and Micromechanics (BioSyM) IRG, Singapore.

\section{References}

1. Siegel, A. C., Bruzewicz, D. A., Weibel, D. B., \& Whitesides, G. M. (2007). Microsolidics: Fabrication of Three-Dimensional Metallic Microstructures in Poly(dimethylsiloxane). Adv. Mater., 19, 727-733.

2. Wu, C.Y., Liao, W.H., \& Tung, Y.C. (2011). Integrated ionic liquid-based electrofluidic circuits for pressure sensing within polydimethylsiloxane microfluidic systems. Lab Chip, 11, 1740-1746.

3. Cheng, S., \& Wu, Z.(2010) Microfluidic stretchable RF electronics. Lab Chip, 10, 3227-3243.

4. Rogers, J.A., Jackman, R.J., Whitesides, G.M., Olson, D.L., \& Sweedler, J.V. (1997). Using microcontact printing to fabricate microcoils on capillaries for high resolution proton nuclear magnetic resonance on nanoliter volumes. Appl. Phys. Lett., 70, 2464-2466.

5. Golda, D., Lang, J.H., \& Culpepper, M.L. (2008). Two-Layer electroplated microcoils with a PECVD silicon dioxide interlayer dielectric. J. Microelectromech. Syst., 17, 1537-1545.

6. Sillerud, L.O., McDowell, A.F., Adolphi, N.L., Serda, R.E., Adams, D.P., Vasile, M.J., \& Alam, T.M. (2006). ${ }^{1} \mathrm{H}$ NMR Detection of superparamagnetic nanoparticles at $1 \mathrm{~T}$ using a microcoil and novel tuning circuit. J. Magn. Reson., 181, 181-190.

7. Kong, T.F., E, H.S., Sugiarto, H.S., Liew, H.F., Wang, X., Lew, W.S., Nguyen, N.T., \& Chen, Y. (2010). An efficient microfluidic sorter: implementation of double meandering micro striplines for magnetic particles switching. Microfluid Nanofluid, 10, 1069-1078.

8. Kong, T.F., Peng, W.K., Luong, T.D., Nguyen, N.T., \& Han, J. (2012). Adhesive-based liquid metal radio-frequency microcoil for magnetic resonance relaxometry measurement. Lab Chip, 12, 287-294.

9. Laser, D.J., \& Santiago, J.G. (2004). A review of micropumps. J. Micromech. Microeng., 14, 35-64.

10. Clark, R.A., Hietpas, P.B., \& Ewing, A.G. (1997). Electrochemical analysis in picoliter microvials. Anal. Chem., 69, 259-263.

11. Grosvenor, A.L., Feltus, A., Conover, R.C., Daunert, S., \& Anderson, K.W. (2000). Development of binding assays in microfabricated picoliter vials: an assay for biotin. Anal. Chem., $72,2590-2594$.

12. Li, P.C.H., \& Harrison, D.J. (1997). Transport, manipulation, and reaction of biological cells on-chip using electrokinetic effects. Anal. Chem., 69, $1564 \mathrm{C} 1568$.

13. Fischbach, F.T., \& Dunning, M.B. (2009). A Manual of Laboratory and Diagnostic Tests. eight edn. Lippincott Williams \& Wilkins.

14. Harder, L., \& Boshkov, L. (2010). The optimal hematocrit. Crit. Care Clin., 26, 335-354.

15. Secomski, W., Nowicki, A., Guidi, F., Tortoli, P., \& Lewin, P.A. (2003). Noninvasive in vivo measurements of hematocrit. J. Ultrasound Med, 22, 375-384.

16. Iftimia, N.V., Hammer, D.X., Bigelow, C.E., Rosen, D.I., Ustun, T., Ferrante, A.A., Vu, D., \& Ferguson, R.D. (2006). Toward noninvasive measurement of blood hematocrit using spectral domain low coherence interferometry and retinal tracking. Opt. Express, 14, 33773388.

17. Campbell, S.A. (2001). The science and engineering of microelectronics fabrication. second edn. Oxford University Press. 